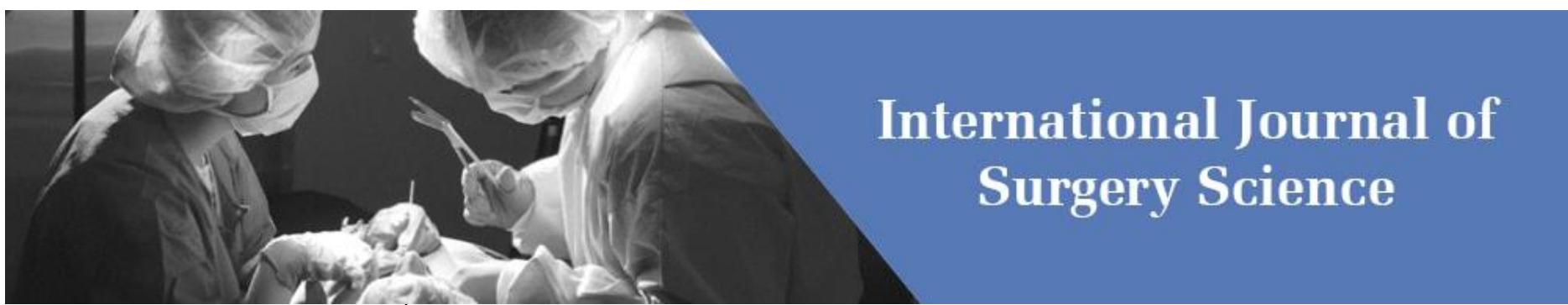

E-ISSN: 2616-3470

P-ISSN: 2616-3462

(C) Surgery Science

www.surgeryscience.com

$2019 ; 3(3): 215-220$

Received: 21-05-2019

Accepted: 23-06-2019

Dr. Dileep Kumar Choudhary

Junior Specialist, Dept. of General

Surgery, Govt. Medical College,

Barmer, Rajasthan, India

Dr. Mahipal Choudhary

Junior Specialist, Dept. of General

Medicine, Govt. Medical College,

Barmer, Rajasthan, India
Correspondence

Dr. Mahipal Choudhary

Junior Specialist, Dept. of General

Medicine, Govt. Medical College,

Barmer, Rajasthan, India

\section{Comparative analysis of different treatment options of grade III and grade IV diabetics foot ulcer to reduce the incidence of amputations}

\author{
Dr. Dileep Kumar Choudhary and Dr. Mahipal Choudhary
}

DOI: https://doi.org/10.33545/surgery.2019.v3.i3d.173

Abstract

Diabetes is fast gaining the status of a potential plague in India with in excess of 62 million diabetic people at present determined to have the illness. At present India beat the world with the most elevated number of individuals with diabetes mellitus. Diabetes is a typical sickness influencing about $14.8 \%$ in provincial and $19.7 \%$ in urban occupants in India. India has most astounding predominance of diabetes in world and records for just about $1 / 6$ of the diabetic patients. This investigation looks at the viability of hyperbaric oxygen treatment, clean dressings, and rhPDGF in grade III and IV diabetic foot ulcers.

Keywords: Diabetic foot, ulcer, conservative treatment, amputation

\section{Introduction}

Diabetes mellitus is a worldwide phenomenon. Type 2 diabetes is the most common form of diabetes in developing countries like India, Africa and China. At present 31.7 million people are diabetic in India. Henceforth, it has been marked as "The diabetic capital of the 1 world". Diabetes warrants a great deal of consideration in light of its different confusions like retinopathy, nephropathy, fringe neuropathy, cardiovascular malady, fringe vascular infection (PVD), cerebrovascular 2 mishaps, hypertension and diabetic foot. Numerous elements like wound, span of diabetes, smoking and deformation can cause foot sore in diabetic patients, anyway fringe vascular ailment (PVD) and neuropathy are the two main considerations that can cause devastation in the foot of the patient. Because of these hazard factors, minor scraped areas which, if, left unattended would 3 be able to cause a ulcer. This ulcer can further get tainted with a range of vigorous and/4 or anaerobic microscopic organisms. Opportune intercession with expansive range anti-infection agents and appropriate diabetic foot care helps in mending the ulcer. This is of most extreme significance, as a deferral would prompt hide the $r$ confusions like removal of foot or appendage. In perspective on the above actualities, a forthcoming report was done to decide the overall recurrence of high-impact and anaerobic microbial separates refined from diabetic foot diseases and to evaluate their near in vitro defenselessness to the ordinarily utilized anti-microbials. Diabetic foot ulcers (DFUs) regularly coincide with vascular deficiency and are the significant reason for gangrene and removal in individuals with diabetes. Danger of creating diabetic foot ulcers is enormously expanded by diminished sensation and blood perfusion. Diabetic foot ulcers speak to a tremendous hazard to the patient's personal satisfaction, heightening wound/disease the board and expenses, and record for an enormous extent of all national medicinal services spending plans ${ }^{[1]}$.

a) Five-year recurrence rates of diabetic foot ulcers are $70 \%$

b) Up to $85 \%$ of all amputations in relation to people with diabetes are preceded by a diabetic foot ulcer

c) People with diabetes with one lower limb amputation have a 50\% risk of developing a serious ulcer in the second limb within 2 years

d) People with diabetes have a $50 \%$ mortality rate in the 5 years following the initial amputation

It is conceivable to decrease removal rates by $49-85 \%$ through a consideration system that consolidates aversion, the interprofessional diabetes care group, suitable association, close checking and instruction. 
Diabetic patients have dependably experienced complexities influencing the lower appendages. Foot disease and the consequent removal of a lower furthest point are most regular reason for hospitalization among diabetic patients. It is increasingly regular in guys between 40 - 60 years old. The sign of diabetic foot issue in our populaces is gross disease and major contributing elements for late introduction incorporate shoeless strolling with anomalous stride, trust in confidence healers and undetected diabetes Ill fitting foot wear prompting foot distortion and ill-advised toe nail cutting expands the hazard while utilization of chappals with a solitary crowd among hallux and second toe results in weight ulcer. Wagner's characterization score might be distinctive for a specialist when contrasted with doctor, since they accompanied propelled malady to a specialist and consequently understanding with grade 0,1 are lesser and those with grade 2,3,4 and 5 are more in our investigation. Fringe neuropathy and disease are regular hazard factors in our investigation ${ }^{[2]}$.

Diabetic patient's face 15-40 times serious danger of removal throughout their lives than do non diabetic people. In Sudan, foot intricacies of diabetes are normal, expensive, and incapacitating. Sound comprehension of the pathogenesis, the range and treatment of the ailment is poor among wellbeing experts. Real lower furthest point removals proceed at the rate of $30-40 \%$ for inpatients in Khartoum's fundamental educating clinic.

The foundation of a Diabetic Foot Center in Khartoum in 1998 set off an enormous deluge of patients with a normal of 80 patients, including 6-8 new cases, coming every day for wound consideration. A few hazard factors that anticipate the result of the diabetic wound have been distinguished by different creators. In any case, not many studys on diabetic foot the executives and result have been accounted for from the African landmass. The target of this investigation was to utilize the wound order criteria embraced by the International Consensus of the Diabetic Foot to anticipate the result with respect to minor and significant lower limit removal. In view of the discoveries of an ongoing, neighborhood ponder, renal disappointment was included as co-horribleness ${ }^{[3]}$.

Foot ulcer is the fundamental introduction of a diabetic foot in our patients $(85 \%)$ in almost $50 \%$ of whom it was gone before by a blister. The unsuitable introductory administration of the blister by patients and wellbeing experts was the principle factor prompting a since a long time ago extended course that would in general end in a removal. A noteworthy worry for the two patients and treating doctors when looked with a diabetic foot is to gauge a result dependent on the accessible information on introduction. We utilized the criteria for wound arrangement received by the International Consensus for the Diabetic Foot to get a dependable evaluating of the diabetic foot and anticipate the result. These criteria were: the level of appendage ischemia, tactile neuropathy, profundity and surface zone of the wound, seriousness of sepsis, and ESRF. Our information are steady with the discoveries of numerous past investigations that have announced that fringe vascular malady in diabetic patients is a free hazard factor for lower appendage removal, without vascular intercession in our arrangement, fringe vascular infection was the most noteworthy sign for MLEA $(\mathrm{P}=0.000)$. Comparable information have been accounted for from other creating nations because of an absence of revascularization administration. The coordinated consideration of basically ischemic diabetic patients by diabetologists, vascular specialists, and podiatrists offers them a helpful major/minor removal proportion $^{[4]}$.
The huge relationship between fringe neuropathy in diabetic patients and lower appendage removal has been archived in numerous past reports. Nonattendance of sensation with the $10 \mathrm{~g}$ monofilament and with $128 \mathrm{~Hz}$ vibration was related with MLEA just as toe removal in patients giving profoundly contaminated neuropathic ulcers with osteomyelitis. Profound and extreme neuroapthic ulcers have been observed to be fundamentally connected with serious neuropathy. Significant removals were likewise prescribed for patients with cutting edge Charcot's arthropathy with serious devastation of the bones and lower leg joints.

A few frameworks of ulcer characterization have been utilized in the writing, the most prevalent one was observed to be Wagner's grouping dependent on six grades of ulcer profundity. Be that as it may, this framework overlooked two significant elements: the level of contamination and the degree of ischemia. The University of Texas San Antonio (UTSA) framework added both contamination and ischemia to the ulcer measurement and subsequently, turned out to be all the more broadly utilized ${ }^{[5]}$

Numerous diabetics with end-arrange renal malady experience the ill effects of cutting edge movement of the infection and experience the ill effects of an amazingly high danger of lower appendage removal. The fortuitous event of fringe vascular malady and fringe neuropathy is undeniably more typical in these patients than in patients without renal disability. Concurrence of fringe vascular infection and average sclerosis is unquestionably progressively basic in the dialysis group. In our investigation populace, we found that real removal rates are altogether higher in patients with end-organize renal illness $(\mathrm{P}=$ 0.003).

Concerning the profundity of the injuries, lower-grade sores reacted well to preservationist treatment with careful debridement and anti-infection agents though those with higher grades required removals. In our study populace, we found that the more profound injuries were altogether connected with toe removal $(P<0.05)$, which could be ascribed to the neighborhood harm of tissues, rot, and osteomyelitis of the phalanges and the metatarsal bones ${ }^{[6]}$.

Disease was a muddling factor in half of our patients who gave foot ulcer $(85 \%)$ and fundamental contamination as fever or surrender was accounted for in $35 \%$ of them. At the point when disease convolutes a diabetic foot, the blend can be appendage or hazardous. The major inclining elements to these contaminations were foot ulceration and different immunological unsettling influences. Oxygen consuming grampositive cocci, particularly Staphylococcus aureus, have been observed to be the prevalent pathogens in diabetic foot diseases. In our study populace, we announced contamination in $63.6 \%$ of the patients with Staphylococcus aureus being the most widely recognized seclude $(33.2 \%)$. Patients with profound sepsis and osteomyelitis (Grade 3) had fundamentally higher rates of toe removals. The mix of osteomyelitis and profound situated sepsis has been observed to be related with $62 \%$ of removals contrasted and 37 and $30 \%$ for every segment autonomously. This is because of profound tissue corruption and nearby ischemia of the toes in view of septic arteritis and thrombosis of computerized corridors.

The rules created by the International Consensus are useful in comprehension the pathophysiology of the diabetic foot and arranging of its treatment. Additionally, the rules are solid prescient elements that decide the result of the administration. To decrease the removal rate, be that as it may, consideration ought to be paid through a multidisciplinary group to opportune referral from the doctor, understanding training, all out contact 
cast, and suitable revascularization. The circumstance is all the more testing in creating nations because of constrained assets with the goal that more pressure ought to be given to aversion, understanding training, and the foundation of multidisciplinary groups in little diabetic units that disperse and apply the global rules on the administration of the diabetic foot ${ }^{[7]}$.

\section{Prevention of Ulcer Formation}

People with diabetes must inspect their feet regularly, or have a family member or care provider do it on their behalf. Daily inspection is the foundation of diabetic foot ulcer prevention. All injuries and wounds ought to be paid attention to right off the bat. Customary, delicate purifying with foamy water, trailed by the use of topical lotions, keeps the skin sound and better ready to oppose breakdown and damage. Shoes ought to be checked to guarantee that they fit appropriately and offer satisfactory help. Think about athletic/sports shoes and thick, cushioned socks. Diabetic socks (unrestrictive on flow) are additionally accessible. On account of foot deformations or uncommon help needs, custom shoes ought to be considered. Minor foot wounds and diseases, for example, cuts, scratches, blisters and tinea pedis (competitor's foot), can be inadvertently intensified by home medications that block healing. Patients ought to be reminded to stay away from hot drenches, warming cushions and cruel topical specialists, for example, hydrogen peroxide, iodine and astringents. Minor injuries ought to be tenderly washed down and treated with topical antiseptics. Moreover, a doctor ought to assess any minor injuries that don't mend rapidly. By strengthening preventive exhortation and assessing the patient's feet at routine subsequent meet-ups, the doctor can enable the patient to create and keep up great foot-care rehearses [8].

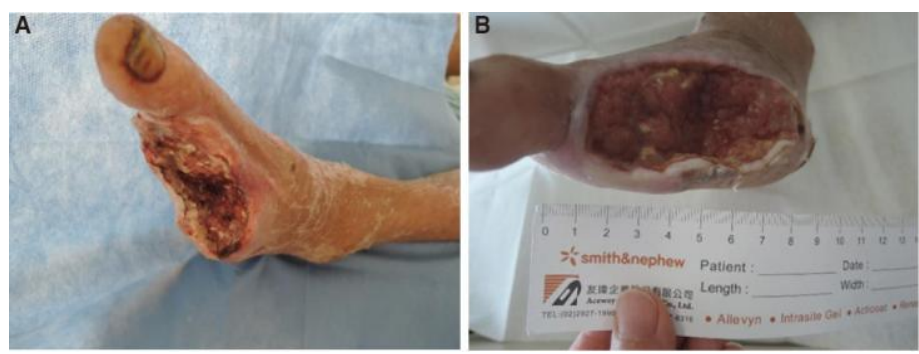

Fig 1: foot deformities and Ulcer Formation

The natural history of a diabetes-related foot ulcer is sobering. The danger of death at 5 years for a patient with a diabetic foot ulcer is 2.5 occasions as high as the hazard for a patient with diabetes who does not have a foot ulcer. The greater part of diabetic ulcers become tainted. Around $20 \%$ of moderate or serious diabetic foot contaminations lead to some degree of removal. Fringe supply route illness autonomously builds the danger of non mending ulcers, contamination, and removal. Mortality after diabetes-related removal surpasses $70 \%$ at 5 years for all patients with diabetes and $74 \%$ at 2 years for that accepting renal-substitution treatment. Regardless of whether such a high mortality is because of a mix of existing together conditions (counting the hazard from a removal strategy), absence of movement, and deconditioning or to different elements isn't clear. The danger of death at 10 years for a patient with diabetes who has had a foot ulcer is twice as high as the hazard for a patient who has not had a foot ulcer. An ongoing appraisal of 785 million outpatient visits by individuals with diabetes in the United States recommended that diabetic foot ulcers and related diseases establish an incredible hazard factor for crisis office visits and medical clinic confirmation. The rate surpasses the rates for congestive heart medical clinic affirmations among patients with diabetes were either for ulcer care or for removal. Thus, the immediate expenses of treating diabetic foot inconveniences surpass the treatment costs for some normal malignant growths. In the United States, a sum of $\$ 176$ billion is spent yearly on direct expenses for diabetes care; as much as $33 \%$ of this consumption is lower-furthest point related, comprising a considerable expense to society. Diabetic foot ulcers are ordinarily brought about by dull worry over a territory that is liable to high vertical or shear worry in patients with fringe neuropathy ${ }^{[9]}$.

\section{Ulcer Healing}

With appropriate therapy surgical debridement, off-loading of pressure, attention to infection, and if necessary, vascular reconstruction foot ulcers heal in many patients, and the need for amputation is averted. On the basis of outcome data in specialized tertiary care hospitals, approximately $77 \%$ of diabetic foot ulcers heal within 1 year. Factors associated with poor healing include advanced end organ disease (congestive heart failure, peripheral artery disease, or end-stage kidney disease requiring renal-replacement therapy) and the inability to walk independently.

\section{Comparative Analysis}

In this study, we have thought about platelet determined development factor (PDGF) gel with Antiseptic dressings and hyperbaric oxygen treatment. PDGF comprises of a group of development elements comprising of two polypeptide chains (An and $B$ ) which structures the dimers, or protein sets: PDGF (AA, AB, BB). Recombinant human (rh) PDGFBB is the main topical development factor to be endorsed with the end goal of wound mending. It has a place with chosen group of items that guarantee to improve twisted healing by expanding the frequency of wound conclusion. Distributed writing demonstrates that every day utilization of rhPDGF has just irrelevant fundamental ingestion. PDGF intervenes tissue fix by means of ${ }^{[10]}$ :

1. mitosis of mesenchymal cells including dermal fibroblasts, smooth muscle cells and wound capillary endothelial cells (angiogenesis);

2. chemoattraction of fibroblasts, smooth muscle cells, monocytes and neutrophils;

3. induction of extracellular matrix components in fibroblasts, including fibronectin and hyaluronic acid;

4. induction of metalloproteinases involved in wound remodeling.

Hyperbaric oxygen (HBO) treatment is characterized as discontinuous organization of $100 \%$ oxygen breathed in at weight more greater than ocean level. The system might be actualized in a stroll in multiplace chamber compacted to profundity with air while the individual inhales $100 \%$ oxygen through head tent, face veil, or endotracheal tube. Multiplace chambers oblige up to six patients at any given moment; every patient is given an individual breathing source. On the other hand the patient might be treated in one individual monoplace chamber pressurized to profundity with oxygen. In either case, the blood vessel weight of oxygen will approach $1500 \mathrm{~mm} \mathrm{Hg}$ at what might be compared to environments outright. Hyperbaric oxygen given as such, additionally alluded to as foundational 
hyperbaric treatment ought not be mistaken for topical oxygen treatment (in appendage encasing gadgets) or unadulterated oxygen breathed in at surrounding climatic weight ${ }^{[11]}$.

\section{Group 1: antiseptic dressings}

Patients belonging to Group 1 were surgically debrided at their initial visit and then treated with following agents ${ }^{[12]}$ :

- EUSOL: Patient's foot was immersed in freshly prepared EUSOL solution for half an hour. Fresh preparation of this solution in standard concentrations was made daily in the wards.

- For the patients who were treated on outpatient basis commercially available EUSOL was used.

- Following treatment with EUSOL, hydrogen peroxide (H2O2) was used on the patient's foot followed by povidone iodine. The ulcer was then dressed with saline gauze followed by secondary dressing.

- Dressing was opened after $24 \mathrm{~h}$ and it was repeated. In this group as well efficacy was measured by $\%$ of ulcers with complete healing at the end of the treatment schedule.

\section{Group 2: hyperbaric oxygen therapy}

Patients in this group were taken for HBO treatment at 2.5 ATA for $60 \mathrm{~min}$ for every sitting for an aggregate of 30 sittings or till the ulcer mended. These sittings were circulated over a time of 10 weeks. Patients were given either every day or exchange day treatment relying upon the accessibility of space in the office. The patients in this group were additionally debrided every once in a while however dressed uniquely with typical saline. No cleaning agents were utilized ${ }^{[13]}$.

\section{Group 3: platelet determined growth factor therapy}

The patients in this group were at first disparaged carefully and in this way just as and when required. The ulcer was treated with day by day nearby use of monetarily accessible PDGF gel. The patients were at first pursued every day by requesting that they visit the clinic day by day. As the ulcer improved with time, or the patient and orderlies took in the right strategy for application; the visits were decreased. The portion of the gel was reconsidered at ordinary interims. The proposed portion for topical application is around $7 \mu \mathrm{g} / \mathrm{cm}^{2}$ of ulcer every day for an individual with normal load of $50 \mathrm{~kg}$. The measure of gel to be connected likewise fluctuates relying on the size of the ulcer. The rough length of gel to be crushed out from the tube=greatest length of the ulcer $x$ most noteworthy width of the ulcer in inches or centimeters duplicated or separated individually by and factor ${ }^{[14]}$.

Table 1: Analysis of results

\begin{tabular}{|lccc|}
\hline Variables & Group I & Group II & Group III \\
No. & 20 & 20 & 20 \\
Mean age (years) & $45=7.574$ & $43.8=9.4$ & $43.35=8.1$ \\
\hline SEM & 1.694 & 2.1 & 1.8 \\
Gender & Male $=11$ & Male $=10$ & Male=11 \\
\hline Mean ulcer healing time (weeks) & $6.75=2.65$ & $6.83=2.5$ & $7.6=2.53$ \\
\% of ulcers showing complete healing & 40 & 60 & 80 \\
\hline Mean ulcer size group & $9.90=5.593 \mathrm{~cm}^{2}$ & $14.91=6.23 \mathrm{~cm}^{2}$ & $19.26=11.315 \mathrm{~cm}^{2}$ \\
No. of patients lost to follow up & $6(30 \%)$ & $5(25 \%)$ & $1(5 \%)$ \\
\hline
\end{tabular}

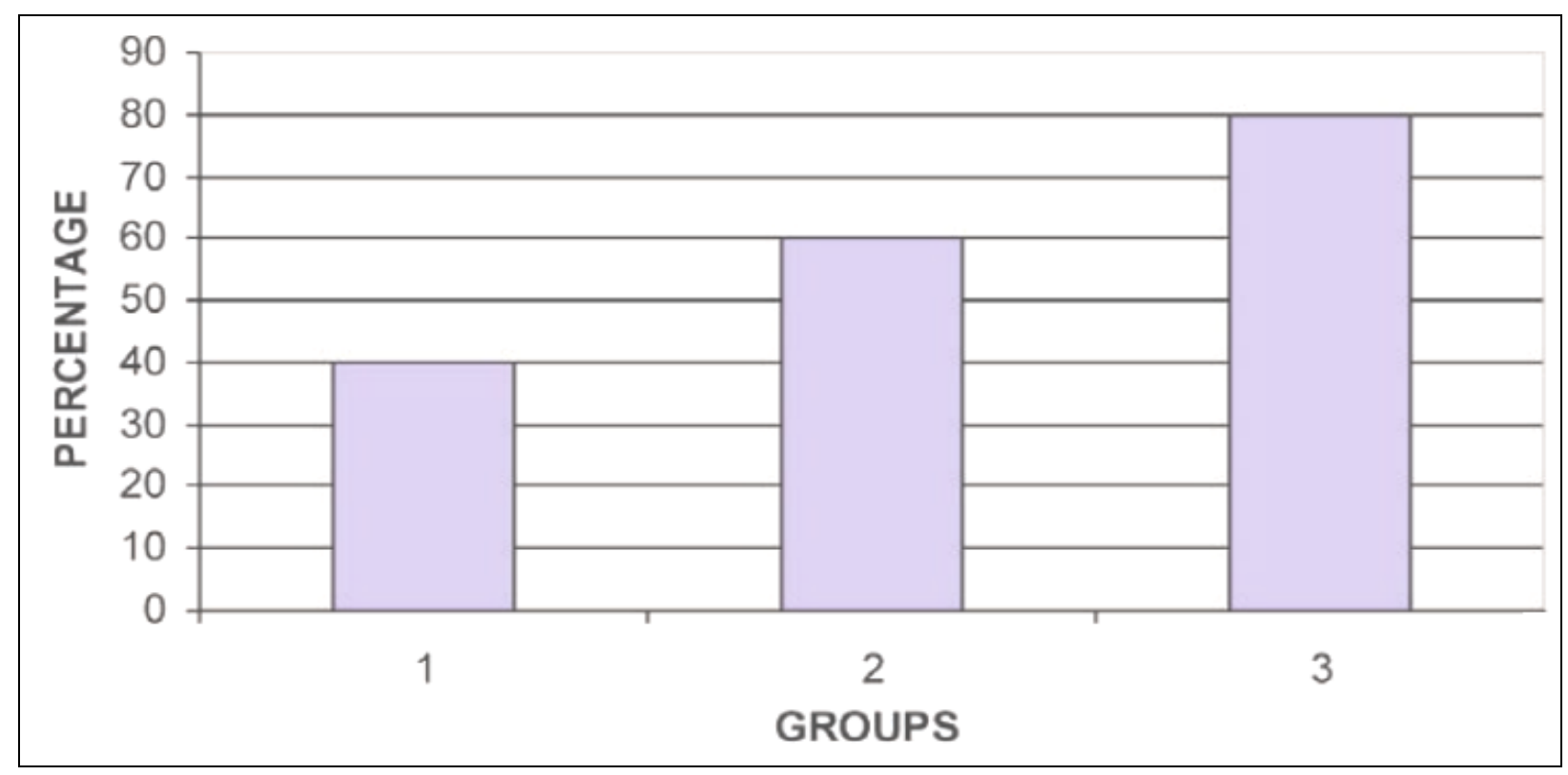

Fig 2: Patients (\%) with complete healing 


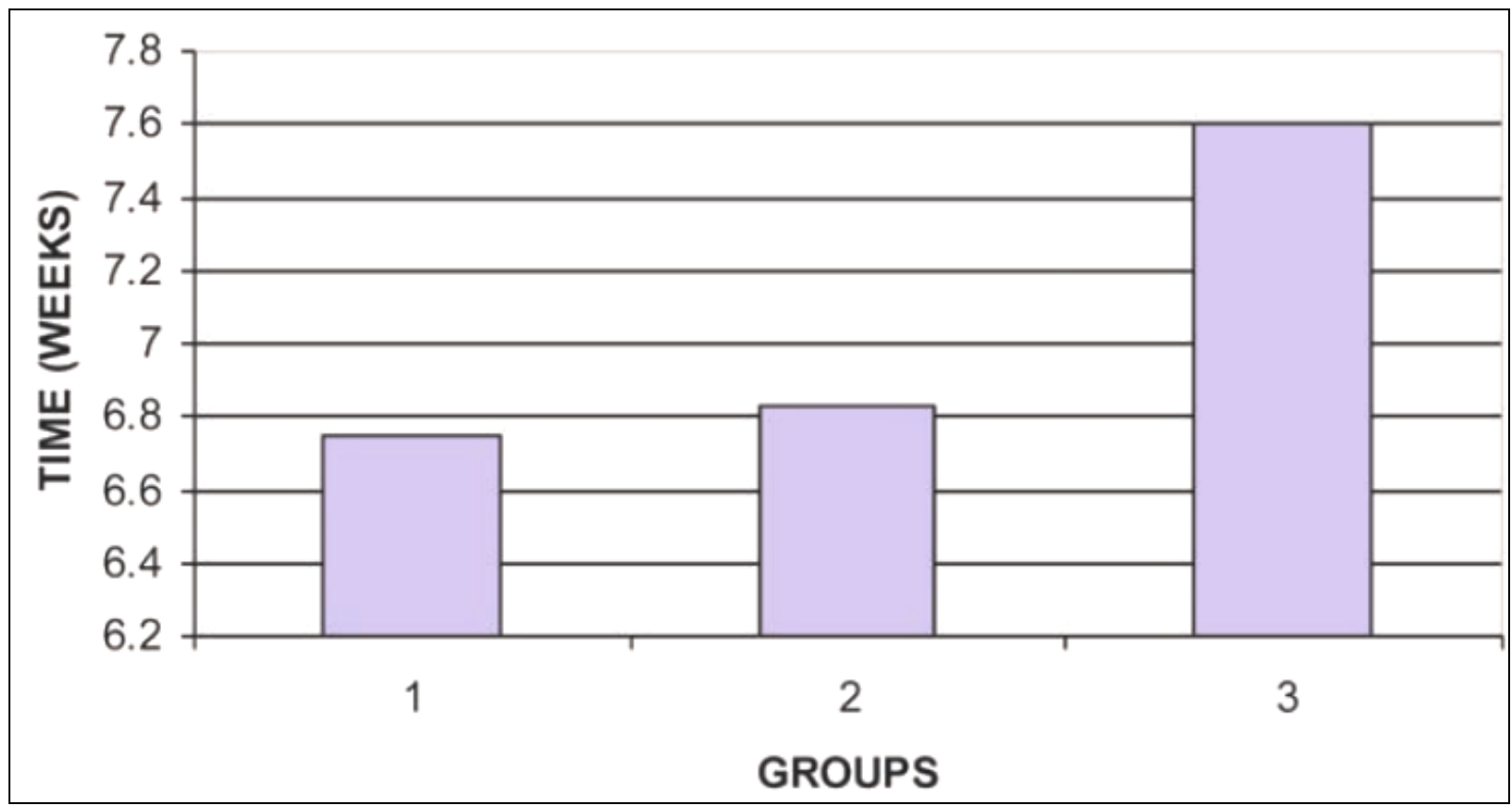

Fig 3: Mean ulcer healing time (weeks)

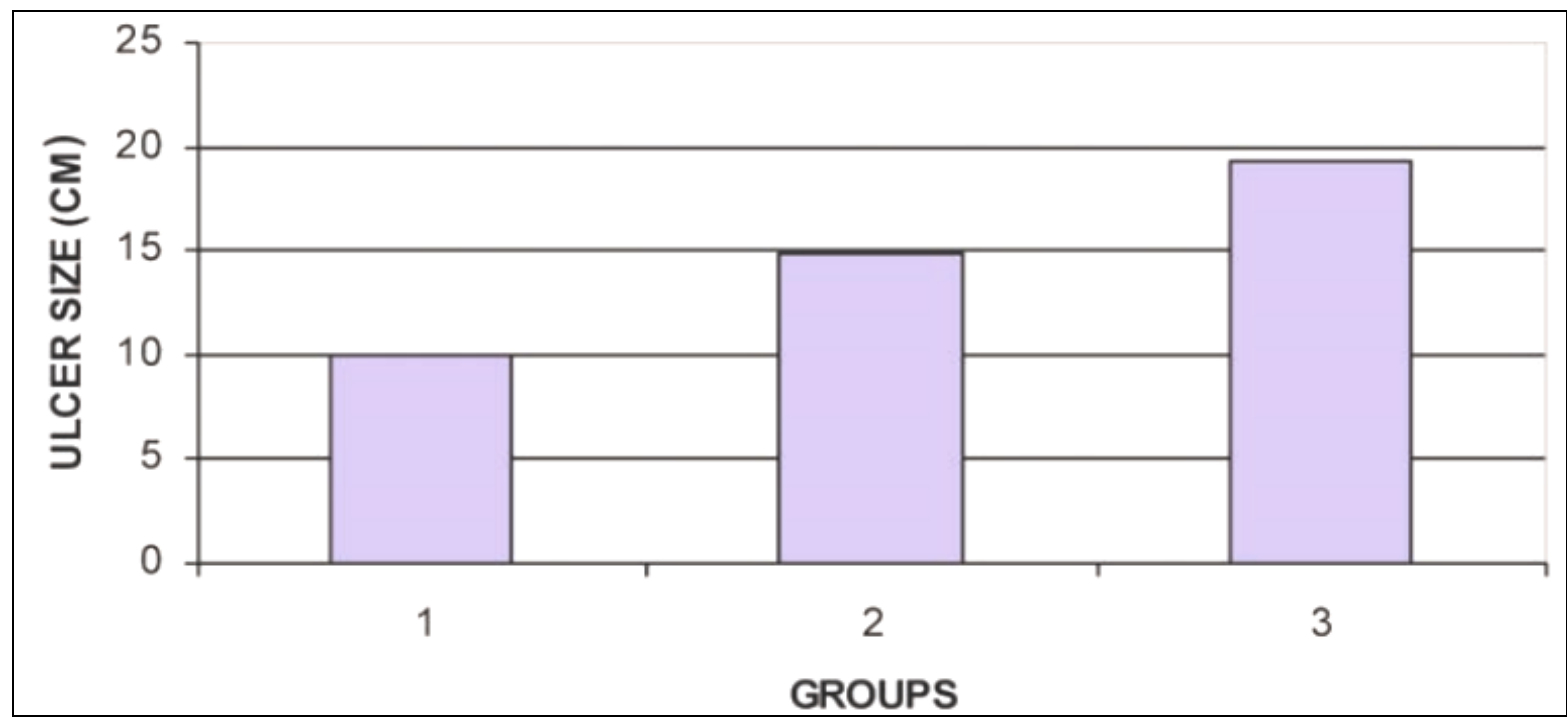

Fig 4: Mean ulcer size $(\mathrm{cm})$.

As we thought about the groups utilizing $\%$ of patients with complete wound contraction, $\mathrm{P}$ worth turns out to be 0.0348 , which is huge. Complete mending\% of rhPDGF (80\%) was essentially higher than HBO therapy $(60 \%)$ which is again fundamentally higher than those of antiseptic dressings $(40 \%)$. In every one of the groups, on contrasting ulcer measure, the $\mathrm{P}$ worth was observed to be 0.0593 , which isn't noteworthy $(>0.05)$. All the equivalent, we would call it not excessively noteworthy on the grounds that it is extremely near 0.05 . Again a greater size of the study group would have given a more clear picture. In this on contrasting rhPDGF and Antiseptic dressings $\mathrm{p}$ worth turned out to be 0.0393 which is really critical and this could one reason for longer healing time in PDGF group ${ }^{[15]}$.

\section{Conclusions}

Diabetic foot ulcer executive requires multidisciplinary and forceful methodology. PDGF ought to be suggested for all grade III and IV diabetic foot ulcer at any rate two months old. HBO is similarly great a choice however has every one of the constraints and symptoms as referenced. Further examinations should be done to demonstrate the predominance of PDGF over HBO or the other way around. Antiseptic operators ought to be maintained a strategic distance from if conceivable utilized for treating diabetic foot ulcers.

\section{References}

1. Park's text book of preventive and social medicine, 22nd edition, 362-365.

2. Diabetes metab Res Rev 2008; 24(1):S7-S13

3. Park text book of preventive and social medicine, edition, $\mathrm{m} / \mathrm{s}$ banarasidas bhanot publication. 2007, 19

4. diabetes mellitus, 14 edition, lippincott Williams and Wilkins publication, chapter. 2006; 67:1123

5. Del Aguila MA, Reiber GE, Koepsell TD. How does provider and patient awareness of high-risk status for lowerextremity amputation influence foot-care practice? Diabetes Care. 1994; 17:1050-1054

6. Andrew JM, Bpoulton, Vileikyte L. Diabetic foot problems and their management around the world. Levin o neal "the diabetic foot" 6th edition mosby, inc 2001; 266. J ayub med 
coll Abbottabad. 2003; 15(3):39

7. Kumar S, Ashe HA, Parnell LN et al. The prevalence of foot ulceration and its correlates in type 2 diabetic patients: A population based study. Diabetic Med. 1994; 11:480-4. JPMI 2012; 26(04):402-407

8. Pecoraro RE, Reiber G, Burgess EM. Pathways to diabetic limb amputation: basis for prevention. Diabetes Care. 1990; 13:513.

9. Apelqvist J, Agardh CD. The association between clinical risk factors and outcome of diabetic foot ulcers. Diabetes Res Clin Pract. 1992; 18:43-53.

10. Chaturvedi N, Stevens LK, Fuller JH, Lee ET, Lu M. Risk factors, ethnic differences and mortality associated with lower-extremity gangrene and amputation in diabetes: The WHO Multinational Study of Vascular Disease in Diabetes. Diabetologia. 2001; 44:65-71.

11. Abbas ZG, Archibald LK. Epidemiology of the diabetic foot in Africa. Med Sci Monit. 2005; 11:RA262-70.

12. International consensus on the diabetic foot and practical guidelines on the management and prevention of the diabetic foot by the international working group on the diabetic foot International working group on the diabetic foot/ consultative section of IDF.

13. Mohammed $\mathrm{H}$ Ahmed. Foot problems in diabetic patients with renal failure. A thesis submitted in partial fulfillment for the requirements of Clinical MD in Surgery of the University of Khartoum, 2004.

14. Adler AI, Boyko EJ, Ahroni JH, Smith DG. Lowerextremity amputation in diabetes: The independent effects of peripheral vascular disease, sensory neuropathy, and foot ulcers. Diabetes Care. 1999; 22:1029-35.

15. Lehto S, Ronnemaa T, Pyorala K, Laakso M. Risk factors predicting lower extremity amputations in patients with NIDDM. Diabetes Care. 1996; 19:607-12. 